\title{
Algorithms for Fast Digital Straight Segments Union
}

\author{
Isabelle Sivignon* \\ Gipsa-lab, CNRS, UMR 5216, F-38420, France \\ isabelle.sivignon@gipsa-lab.grenoble-inp.fr
}

\begin{abstract}
Given two Digital Straight Segments (DSS for short) of known minimal characteristics, we investigate the union of these DSSs: is it still a DSS ? If yes, what are its minimal characteristics ? We show that the problem is actually easy and can be solved in, at worst, logarithmic time using a state-of-the-art algorithm. We moreover propose a new algorithm of logarithmic worst-case complexity based on arithmetical properties. But when the two DSSs are connected, the time complexity of this algorithm is lowered to $\mathcal{O}(1)$ and experiments show that it outperforms the state-of-the art one in any case.
\end{abstract}

Keywords: Digital geometry, Union, Digital straight segment.

\section{Introduction}

Digital Straight Lines (DSL) and Digital Straight Segments (DSS) have been used for many years in many pattern recognition applications involving digital curves. Whether it be for polygonal approximation or to design efficient and precise geometric estimators, a basic task is the so-called DSS recognition problem: given a set of pixels, decide whether this set is a DSS and compute its characteristics. Many linear-in-time algorithms have been proposed to solve this problem through the years. Furthermore, Constructive Solid Geometry-like operations have been considered for these objects: the intersection of two DSL has been studied in [151685], algorithms for the fast computation of subsegments were described in 917. Surprisingly enough, the union of DSSs has not yet been studied. The problem was raised in [2] in the context of parallel recognition of DSSs along digital contours. The recognition step was followed by a merging step where the problem of DSSs union appeared. In this work, we show how to solve this problem, both using state-of-the-art algorithm, and proposing a new and faster algorithm.

\footnotetext{
* This work was partially founded by the French Agence Nationale de la Recherche (Grant agreement ANR-11-BS02-009).
} 


\section{General Considerations}

\subsection{Preliminary Definitions}

A Digital Straight Line (DSL for short) of integer characteristics $(a, b, \mu)$ is the infinite set of digital points $(x, y) \in \mathbb{Z}^{2}$ such that $0 \leq a x-b y+\mu<\max (|a|,|b|)$ 4. These DSL are 8-connected and often called naive. The fraction $\frac{a}{b}$ is the slope of the DSL, and $\frac{\mu}{b}$ is the shift at the origin. In the following, without loss of generality, we assume that $0 \leq a \leq b$. The remainder of a DSL of characteristics $(a, b, \mu)$ for a given digital point $(x, y)$ is the value $a x-b y+\mu$. The upper (resp. lower) leaning line of a DSL is the straight line $a x-b y+\mu=0$ (resp. $a x-b y+\mu=b-1$ ). Upper (resp. lower) leaning points are the digital points of the DSL lying on the upper (resp. lower) leaning lines. A Digital Straight Segment (DSS) is a finite 8-connected part of a DSL.

If we consider the digitisation process related to this DSL definition, the points of the DSL $\mathbf{L}$ of parameters $(a, b, \mu)$ are simply the grid points $(x, y)$ lying below or on the straight line $l: a x-b y+\mu=0$ (Object Boundary Quantization), and such that the points $(x, y+1)$ lie above $l$. Otherwise said, line $l$ separates the points $X$ of the DSL from the points $X+(0,1)$ [14, and is called separating line. More generally, for an arbitrary set of digital points $X$, the separating lines are the lines that separate the points $X$ from the points $X+(0,1)$. In other words, the separating lines separate the upper convex hull of $X$ from the lower convex hull of $X+(0,1)$. Computing the set of separating lines of two polygons is a very classical problem of computational geometry. It is well known that specific lines called critical support lines can be defined: there are the separating lines passing through a point of each polygon boundary. Critical support points are the points of the polygons belonging to critical support lines [12].

All the separating lines of a DSL have the same slope, but this is not true for arbitrary sets of digital points. The minimal characteristics of a set of digital points $X$ are the characteristics of the separating line of minimal $b$ and minimal $\mu$. The set of separating lines of a DSS is well known, and the critical support points are exactly defined by the DSS leaning points: they define the minimal characteristics of the DSS.

The set of separating lines of a set of points $X$ can also conveniently be defined in a dual space, also called parameter space. In this space a straight line $l: \alpha x-y+\beta=0$ is represented by the 2D point $(\alpha, \beta)$. Given a set of digital points $X$, a line $l: \alpha x-y+\beta=0$ is a separating line if and only if for all $(x, y) \in X, 0 \leq \alpha x-y+\beta<1$. This definition is strictly equivalent to the one given previously. The preimage of $X$ is the representation of its separating lines in the dual space and is defined as $\mathcal{P}(X)=\{(\alpha, \beta), 0 \leq \alpha \leq 1,0 \leq \beta \leq$ 1| $\forall(x, y) \in X, 0 \leq \alpha x-y+\beta<1\}$. The set of separating lines of a set of pixels is an open set in the digital space, but it is a convex polygon in the dual space. In this work, this dual space will not be used explicitly in the algorithms, but we will see that this representation is convenient in some proofs. Moreover, the arrangement of all the constraints for any pixel $(x, y)$ with $y \leq x \leq n$ is called Farey Fan [10] of order $n$ : each cell of this arrangement is the preimage of a DSS 
of length $n$. Figure 1 is an illustration of the separating lines of a DSS, both in the digital space and in the dual space: they separate the points $X$ of the DSS in black, from the points $X+(0,1)$ in white. Note that the edges of the preimage of a DSS are exactly supported by the dual representation of its leaning points, or equivalently its critical support points.

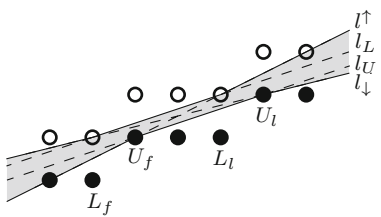

(a)

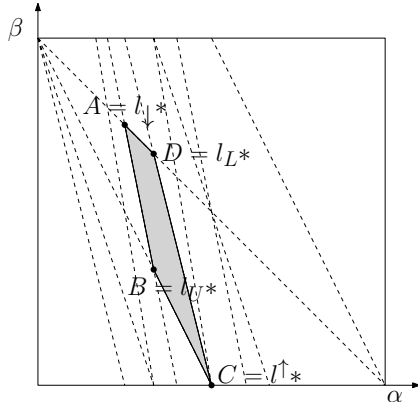

(b)

Fig. 1. (a) DSS of minimal characteristics $(1,3,1)$ with its leaning points $U_{f}, U_{l}, L_{f}, L_{l}$. (b) Each vertex of the preimage maps to a straight line in the digital space. The vertex $B\left(\frac{1}{3}, \frac{1}{3}\right)$ maps to the upper leaning line, the characteristics of which are the minimal characteristics of the DSS.

\subsection{Setting the Problem and Useful Properties}

Consider now the following problem :

Problem 1. Given two DSSs $S_{1}=\left[P_{1} Q_{1}\right]$ and $S_{2}=\left[P_{2} Q_{2}\right]$ of known minimal characteristics, decide if there exists a DSL containing both $S_{1}$ and $S_{2}$. If yes, compute the minimal characteristics of $S_{1} \cup S_{2}$.

If $S_{1}$ and $S_{2}$ do not belong to the same octant, then it is easy to conclude that there is no DSL containing both $S_{1}$ and $S_{2}$. Thus, in the following $S_{1}$ and $S_{2}$ belong to the first octant, i.e. we have $S_{1}\left(a_{1}, b_{1}, \mu_{1}\right)$ and $S_{2}\left(a_{2}, b_{2}, \mu_{2}\right)$ with $0 \leq a_{1} \leq b_{1}$ and $0 \leq a_{2} \leq b_{2}$. We denote by $r_{i}(x, y)=a_{i} x-b_{i} y+\mu_{i}, i \in\{1,2\}$ the remainder function of each DSS.

In what follows, we moreover suppose that the leaning points of $S_{1}$ and $S_{2}$ are known as input data. This is not a strong requirement since the most efficient recognition algorithms actually compute this data on the fly.

By convention, we also suppose that the abscissa of $Q_{2}$ is greater than the abscissa of $Q_{1}$. Note that we make no assumption on the connectivity of $S_{1}$ and $S_{2}$ : the abscissa of $P_{2}$ can be lower than, equal to or greater than the abscissa of $Q_{1}$. If the abscissa of $P_{2}$ is lower than the abscissa of $P_{1}$, then the problem is trivial since $S_{1}$ is either a subsegment of $S_{2}$ or the union is impossible. 
Consequently, we also assume that the abscissa of $P_{2}$ is greater than the abscissa of $P_{1}$.

The first part of Problem 1 consists in deciding if there exists a separating line for the set $S_{1} \cup S_{2}$ : we will say that the union is possible in this case. If so, then among all the separating lines, the final goal is to find the one with minimal characteristics.

Property 1. The preimage of $S_{1} \cup S_{2}$ is equal to the intersection between the preimages of $S_{1}$ and $S_{2}$.

Proof. The proof is straightforward since the lines that are separating for $S_{1} \cup S_{2}$ are the ones that are separating for $S_{1}$ and $S_{2}$.

Corollary 1. The critical support points of the set of separating lines of $S_{1} \cup S_{2}$ are either upper leaning points or lower leaning points translated by $(0,1)$ of $S_{1}$ and $S_{2}$. Thus, to compute the set of separating lines of $S_{1} \cup S_{2}$, it is enough to update the set of separating lines of $S_{1}$ with the leaning points of $S_{2}$ (or conversely).

Proof. The critical support points are, in the dual space, lines supporting the edges of the preimage. From Property 1 the lines supportting the edges of $\mathcal{P}\left(S_{1} \cup\right.$ $S_{2}$ ) are lines supporting the edges of $\mathcal{P}\left(S_{1}\right)$ or $\mathcal{P}\left(S_{2}\right)$. However, since $S_{1}$ and $S_{2}$ are DSSs, the edges of their preimages are supported by the dual representation of either upper leaning points or lower leaning points translated by $(0,1)$.

\section{Fast Union of DSSs: An Arithmetical Algorithm}

\subsection{Fast Computation of the Set of Separating Lines}

A first straightforward solution to compute the set of separating lines of $S_{1} \cup$ $S_{2}$ is to use the state-of-the-art algorithm of O'Rourke [11, re-interpreted in the digital space by Roussillon [14. Whether it be in the dual space or in the digital space, these algorithms update the critical support points iteratively for each point added. Since at most four points have to be considered in our case, the algorithm is already quite efficient compared to the classical arithmetical recognition algorithm for instance. However, we propose an algorithm that is both faster and simpler to implement, in the spirit of the arithmetical recognition algorithm.

The idea is the following: if we know that the slopes of the separating lines of $S_{1} \cup S_{2}$ are greater/lower than the slopes (given by the minimal characteristics) of $S_{1}$ and $S_{2}$ respectively, then we can conclude that some leaning points of $S_{1}$ or $S_{2}$ cannot be critical support points for $S_{1} \cup S_{2}$.

Property 2. Let $S_{1}$ be a DSS of minimal characteristics $\left(a_{1}, b_{1}, \mu_{1}\right)$. Let $L_{1 f}, L_{1 l}$, $U_{1 f}, U_{1 l}$ be its first and last, lower and upper leaning points. If all the separating lines of $S_{1} \cup S_{2}$ have a slope greater (res. lower) than $\frac{a_{1}}{b_{1}}$, then $U_{1 l}$ (resp. $U_{1 f}$ ) and $L_{1 f}$ (resp. $L_{1 l}$ ) are not critical support points for $S_{1} \cup S_{2}$. 
Proof. If all the separating lines of $S_{1} \cup S_{2}$ have a slope lower than $\frac{a_{1}}{b_{1}}$, then, in the dual space and from Property 1, $\mathcal{P}\left(S_{1} \cup S_{2}\right)$ is a subpart of the triangle defined by the vertices $A B D$ (see Figure [1(b)). In particular, the edges $[B C]$ and $[D C]$ of $\mathcal{P}\left(S_{1}\right)$ supported by $U_{1 f}^{*}$ and $L_{1 l}^{*}$ respectively cannot be edges of $\mathcal{P}\left(S_{1} \cup S_{2}\right)$. Therefore, the leaning points $U_{1 f}$ and $L_{1 l}$ are not critical support points for $S_{1} \cup S_{2}$. The proof is the same if we suppose that the separating lines all have a slope greater than $\frac{a_{1}}{b_{1}}$.

Note that if $S_{1}$ has three leaning points only, let's say for instance only one lower leaning point $L_{1}$, then setting $L_{1 l}$ and $L_{1 f}$ to $L_{1}$ ( $L_{1}$ is "duplicated"), the property is also valid. A similar result holds when the leaning points of $S_{2}$ are considered. However, guessing the slope of the union can be tricky, and taking into account only the DSS slopes is not enough. For example, it is easy to exhibit cases where the slope of $S_{2}$ is greater than the slope of $S_{1}$, and the slope of $S_{1} \cup S_{2}$ is nevertheless lower than both the slope of $S_{1}$ and the slope of $S_{2}$ (see Figure 2(a)). We establish hereafter some properties linking the remainder of the leaning points of $S_{2}$ and the slope of the separating lines for $S_{1} \cup S_{2}$ if they exist.

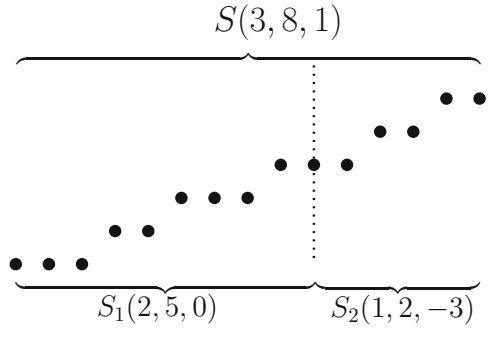

(a)

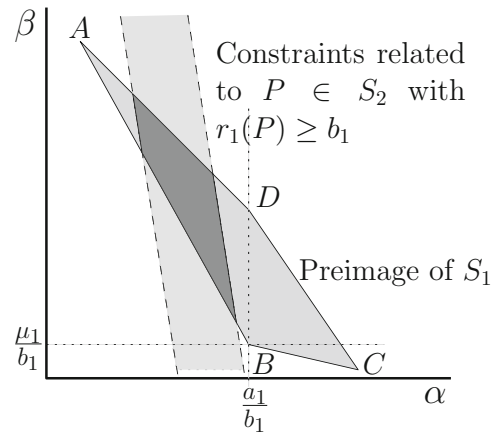

(b)

Fig. 2. (a) The slope of $S_{1}$ is equal to $\frac{2}{5}$ and lower than the slope of $S_{2}$, which is equal to $\frac{1}{2}$. However, the slope of $S_{1} \cup S_{2}$ is equal to $\frac{3}{8}$ and smaller than both. (b) Illustration of Property 4.

Let's start with a very simple consideration.

Property 3. If for all the leaning points $P$ of $S_{2}$ (resp. $S_{1}$ ), we have $0 \leq r_{1}(P)<$ $b_{1}$ (resp. $0 \leq r_{2}(P)<b_{2}$ ), then there exists a DSL containing $S_{1} \cup S_{2}$ and its minimal characteristics are the one of $S_{1}\left(\operatorname{resp} . S_{2}\right)$.

Proof. In the dual space, the points $B$ and $D$ of the preimage of $S_{1}$ satisfy all the constraints related to the points $P$ defined as above, which ends the proof.

With the following property, we investigate the other cases. 
Property 4. Let $P$ be a leaning point of $S_{2}$.

- if $\mathbf{r}_{\mathbf{1}}(\mathbf{P}) \geq \mathbf{b}_{\mathbf{1}}$, the slope of all the separating lines for $S_{1} \cup S_{2}$, if any, is lower than the slope of $S_{1}$

- if $\mathbf{r}_{\mathbf{1}}(\mathbf{P})<\mathbf{0}$, the slope of all the separating lines for $S_{1} \cup S_{2}$, if any, is greater than the slope of $S_{1}$

Let $P$ be a leaning point of $S_{1}$.

- if $\mathbf{r}_{\mathbf{2}}(\mathbf{P}) \geq \mathbf{b}_{\mathbf{2}}$, the slope of all the separating lines for $S_{1} \cup S_{2}$, if any, is greater than the slope of $S_{2}$

- if $\mathbf{r}_{\mathbf{2}}(\mathbf{P})<\mathbf{0}$, the slope of all the separating lines for $S_{1} \cup S_{2}$, if any, is lower than the slope of $S_{2}$

Proof. We prove the first item, for a leaning point of $S_{2}$ with a remainder greater than or equal to $b_{1}$. Proving the other cases is similar. Consider a point $P \in S_{2}$ such that $r_{1}(P) \geq b_{1}$. Let us consider the stripe defined by the constraints related to this point in the dual space. It is very simple to see that the point $\left(\frac{a_{1}}{b_{1}}, \frac{\mu_{1}}{b_{1}}\right)$ is above this stripe (see Figure 2(b)). Since $P$ does not belong to $S_{1}$, and with the assumptions made in Section 2.2 on the relative position of $S_{1}$ and $S_{2}$, its abscissa is greater than the abscissas of all the leaning points of $S_{1}$. This means that the intersection, if not empty, between the stripe and $\mathcal{P}\left(S_{1}\right)$ lies in the subspace $\alpha<\frac{a_{1}}{b_{1}}$.

Table 1 summarises the computation of the four possible critical support points combining Properties 2 and 4. Figure 6] in Appendix illustrates the first line of this table.

Table 1. Possible critical support points according to remainder values

\begin{tabular}{|c|c|c|c|}
\hline & value & $<0$ & $\geq b$ \\
\hline$P \in S_{2}$ & $r_{1}(P)$ & $\left(U_{f}, L_{f}\right)=\left(U_{1 f}, L_{1 l}\right)$ & $\left(U_{f}, L_{f}\right)=\left(U_{1 l}, L_{1 f}\right)$ \\
\hline$P \in S_{1}$ & $r_{2}(P)$ & $\left(U_{l}, L_{l}\right)=\left(U_{2 l}, L_{2 f}\right)$ & $\left(U_{l}, L_{l}\right)=\left(U_{2 f}, L_{2 l}\right)$ \\
\hline
\end{tabular}

At this point, we have identified four points denoted by $U_{f}, U_{l}, L_{f}$ and $L_{l}$, that may be critical support points for $S_{1} \cup S_{2}$. However, they may not be all critical support points. Since the preimage of $S_{1} \cup S_{2}$ is a convex polygon, it has at least three edges and thus, at least three out of the four possible points are indeed critical support points. Property 5 gives a way to decide whether the four points are critical support points or not.

Property 5. $U_{f}$ and $U_{l}$ (resp. $L_{f}$ and $L_{l}$ ) are both critical support points if and only if $L_{f}$ and $L_{l}$ (resp. $U_{f}$ and $U_{l}$ ) belong to the DSL of directional vector $U_{l}-U_{f}$ (resp. $L_{l}-L_{f}$ ) and upper (resp. lower) leaning points $U_{f}$ and $U_{l}$ (resp. $L_{f}$ and $\left.L_{l}\right)$. 
Proof. $U_{f}$ and $U_{l}$ are both critical support points is equivalent to say that the straight line $\left(U_{f} U_{l}\right)$ is separating for $S_{1} \cup S_{2}$. This is also equivalent to the fact that $L_{f}$ and $L_{l}$ belong to the DSL as defined in the property statement.

If the four points are not all critical support points, the three critical support points are identified using Property 6 .

Property 6. Let $U_{f}, U_{l}, L_{f}$ and $L_{l}$ be the four possible critical support points for $S_{1} \cup S_{2}$. If they are not all critical support points, then:

- if $U_{f}$ and $U_{l}$ are both critical support points then:

- if the slope of $\left(U_{f} U_{l}\right)$ is lower than the slope of $\left(L_{f} L_{l}\right), L_{f}$ is the third critical support point ;

- otherwise, $L_{l}$ is the third critical support point ;

- if $L_{f}$ and $L_{l}$ are both critical support points then:

- if the slope of $\left(L_{f} L_{l}\right)$ is greater than the slope of $\left(U_{f} U_{l}\right), U_{f}$ is the third critical support point ;

- otherwise, $U_{l}$ is the third critical support point ;

Proof. We write the proof for the case where $U_{f}$ and $U_{l}$ are both critical support points. The other case is similar.

Consider the dual representation of the points $U_{f}$ and $U_{l}$, denoted by $U_{f}^{*}$ and $U_{l}^{*}$. By hypothesis, these two lines support edges of $\mathcal{P}\left(S_{1} \cup S_{2}\right)$. Consider now the dual representation of the points $L_{f}+(0,1)$ and $L_{l}+(0,1)$, denoted by $L_{f+}^{*}$ and $L_{l+}^{*}$. The third edge of $\mathcal{P}\left(S_{1} \cup S_{2}\right)$ is a segment of either $L_{f+}^{*}$ or $L_{l+}^{*}$. We suppose now that the slope of $\left(U_{f} U_{l}\right)$ is lower than the slope of $\left(L_{f} L_{l}\right)$ and illustrate the rest of the proof with Figure 3 . Then, the abscissa of point $D=L_{f+}^{*} \cap L_{l+}^{*}$ is greater than the abscissa of point $B=U_{f}^{*} \cap U_{l}^{*}$ (it lies in the light-gray half-space on Figure 3). It is now easy to see that if $D$ is above the line $U_{f}^{*}$, then both $L_{f+}^{*}$ and $L_{l+}^{*}$ support edges of $\mathcal{P}\left(S_{1} \cup S_{2}\right)$, which is not possible by hypothesis. Then, $D$ is below the line $U_{f}^{*}$, which implies that the third edge of the preimage is a segment of $L_{f+}^{*}$, and equivalently $L_{f}$ is the third critical support point.

\subsection{Pulling Out the Minimal Characteristics}

In the previous section, we showed how to efficiently compute the three or four critical support points of $S_{1} \cup S_{2}$. These points also define the preimage of $S_{1} \cup S_{2}$. Until now, the results were valid whether the two DSSs were connected or not. In order to compute the minimal characteristics, we have to consider several cases.

Input DSSs Are Connected. We consider here the case where the first point of $S_{2}$ is either a point of $S_{1}$ or 8-connected to the last point of $S_{1}$. In this case, if there exists a DSL containing $S 1 \cup S_{2}$, then $S_{1} \cup S_{2}$ is a DSS of length $n$, the difference of abscissa between the first point of $S_{1}$ and the last point of $S_{2}$. As a consequence, $\mathcal{P}\left(S_{1} \cup S_{2}\right)$ is a cell of the Farey Fan of order $n$, with very wellknown properties. In particular, the critical support points computed in Section 3.1 are exactly the leaning points of the DSS. 


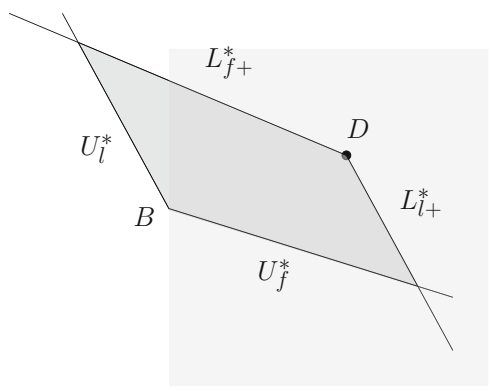

(a)

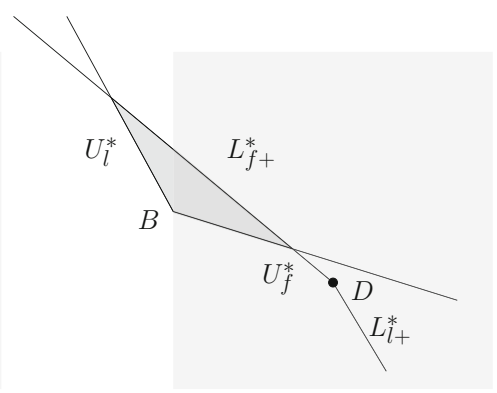

(b)

Fig. 3. Illustration of the proof of Property 6

$S_{1}$ Last Point and $S_{2}$ First Point Have the Same Ordinate. We show that this case is actually as easy as the previous one.

Property \%. If the last point of $S_{1}$ and the first point of $S_{2}$ have the same ordinate, then $\mathcal{P}\left(S_{1} \cup S_{2}\right)$ is a unique cell of the Farey Fan of order $n$, the difference of abscissa between the last point of $S_{2}$ and the first point of $S 1$.

Proof. In a DSS, the edges of the preimage are defined by the leaning points only. Actually, the preimage of a DSS is equal to the preimage of its leaning points, all the other points make no contribution. If the last point of $S_{1}$ and the first point of $S_{2}$ have the same ordinate, then all the missing points between these two points also have this same ordinate. Consequently, they cannot be neither lower nor upper leaning points for any DSL containing $S_{1} \cup S_{2}$. This proves that $\mathcal{P}\left(S_{1} \cup S_{2}\right)$ is the same as the preimage of the set of pixels composed of $S_{1}, S_{2}$ and all the missing points between the two. Then, $\mathcal{P}\left(S_{1} \cup S_{2}\right)$ is the preimage of a DSS of length $n$, the difference of abscissa between the last point of $S_{2}$ and the first point of $S 1$, which is similar to the previous case.

Disconnected DSSs. This case is trickier since $\mathcal{P}\left(S_{1} \cup S_{2}\right)$ may not be a unique cell but can be a union of adjacent cells of a Farey Fan (see Figure 4 for an example). The characteristics given by the critical points may not be the minimal ones. However, from the critical support points we can easily infer the range of slopes of the separating lines. If we denote $s_{\text {low }}$ and $s_{\text {up }}$ the minimum and maximum slopes of the separating lines, the slope of the line of minimal characteristics is given by the fraction of smallest denominator between $s_{l o w}$ and $s_{u p}$. It is finally easy to decide which one of either $U_{f}$ or $U_{l}$ is an upper leaning point of the line of minimal characteristics (see Algorithm 1 for more details).

\subsection{General Algorithm}

All the properties presented above are put together to design the fast union algorithm described in Algorithm [1. The algorithm returns the minimal characteristics of $S_{1} \cup S_{2}$ if the union is possible. The result is given as a directional 


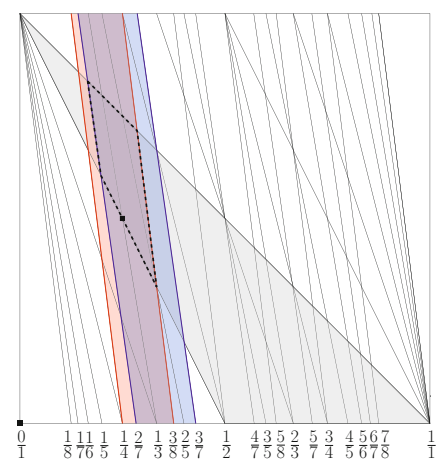

(a)

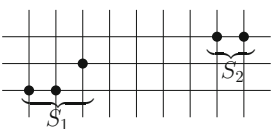

(b)

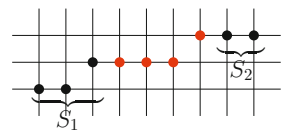

(d)

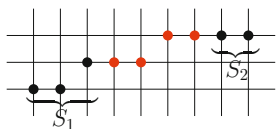

(c)

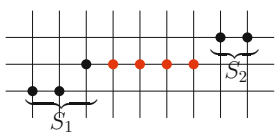

(e)

Fig. 4. When $S_{1}$ and $S_{2}$ are not connected, like the ones depicted in (b), $\mathcal{P}\left(S_{1} \cup S_{2}\right)$ may be the union of several cells: in (a), $\mathcal{P}\left(S_{1}\right)$ is depicted in light gray, and the two constraints related to the leaning points of $S_{2}$ are depicted in red and blue. The intersection is bordered by a dotted black line: it is composed of three cells, each one being the preimage of a DSS containing $S_{1} \cup S_{2}$, depicted in (c), (d), and (e).

vector $(b, a)$ and an upper leaning point. The algorithm can be decomposed into three main parts. Between line 1 and 2, the four possible critical support points are computed. The function initCriticalsupportPoint is the implementation of Table 1 and is detailed in Algorithm 2 presented in Appendix. The string parameter given in input is there to discriminate between the two lines of Table 1. At the same time, easy cases where $S_{1} \cup S_{2}$ has the same minimal characteristics as $S_{1}$ or $S_{2}$ are treated: in such cases, the variable inDSL is set to true by initCriticalSupportPoint and we can conclude directly. Then, between lines 3 and 4, the exact critical support points are computed. The function isSolution? implements Property 5 and is detailed in Algorithm 3 in Appendix: here, the string parameter tells if the upper leaning points are tested w.r.t the lower leaning points or conversely. The function thirdPoint implements Property 6 , if the variable solU is true, then the first item of the property is concerned, otherwise solL is true, and the second item is concerned. The last part, between lines 5 and 7 returns the minimal characteristics of $S_{1} \cup S_{2}$. On line 6 , if $S_{1}$ and $S_{2}$ are connected or if the last point of $S_{1}$ and the first point of $S_{2}$ have the same ordinate, the result is straightforward from the critical support points. Otherwise, as explained in Section 3.2 the line of minimal characteristics is computed among all the separating lines. Function minimalslope is then called. It can be implemented in several way, using for instance the decomposition into continued fractions, or, like in [16]-Algorithm 3 (see also [76]) using the Stern-Brocot tree.

\subsection{Complexity Analysis}

Lemma 1. The complexity of Algorithm 1 is $\mathcal{O}(1)$ when $S_{1}$ and $S_{2}$ are connected or when the last point of $S_{1}$ and the first point of $S_{2}$ have the same ordinate. Its 


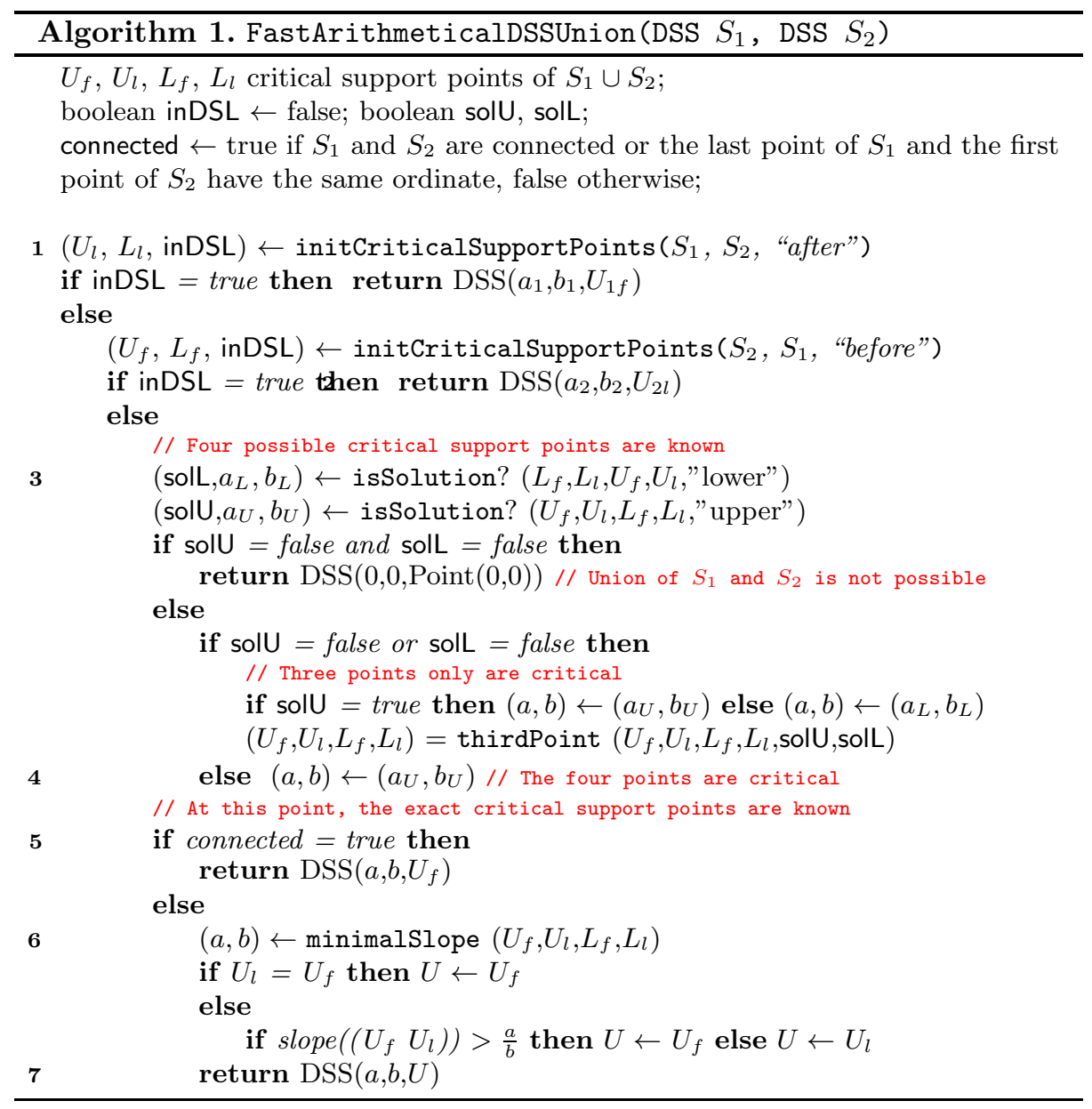

complexity is $\mathcal{O}(\log (n))$ otherwise, where $n$ is the difference of abscissa between the last point of $S_{2}$ and the first point of $S_{1}$.

Proof. If we assume a computing model where the standard arithmetic operations are done in constant time, then all the operations from line 1 to line 5 are also done in constant time. Whichever the algorithm chosen, the function minimalSlope on line 6 always requires, in a more or less direct way, the computation of the continued fractions of two fractions $\frac{p}{q}$ with $p \leq q \leq n$, and $n$ is the difference of abscissa between the last point of $S_{2}$ and the first point of $S_{1}$. This is done in $\mathcal{O}(\log (n))$ time (see [6] for instance). 


\section{Experimental Results}

Algorithm 1 was implemented in C++ using the open-source library DGtal [1. We refer to it as the FastArithmetical algorithm in the following. We compare our algorithm with two other ones. The first one is the well-known arithmetical recognition algorithm [4, implemented in DGtal (called Arithmetical algorithm in what follows). As stated at the beginning of Section 3.1, the algorithm of O'Rourke [11] can be used to compute the set of separating lines. It was implemented in DGtal by T. Roussillon as the StabbingLine algorithm. The Arithmetical algorithm works only when the two DSSs are connected and is used as follows : the minimal characteristics are initialised with the ones of $S_{1}$, and updated as the points of $S_{2}$ are added one by one. Concerning the StabbingLine algorithm, the preimage is initialised with the one of $S_{1}$ and updated as the leaning points of $S_{2}$ are added (Corollary 11). The result is the set of critical support points of $S_{1} \cup S_{2}$.

The experimental setup is the following:

- a DSL of characteristics $(a, b, \mu)$ is picked up at random ;

- the abscissas $x_{1}$ and $x_{2}$ of the first and last points of $S_{1}$ are randomly selected ;

- the abscissa $x_{3}$ of the first point of $S_{2}$ is either equal to $x_{2}+1$ in the connected case, or randomly selected and greater than $x_{2}$ in the disconnected one ;

- the abscissa of the last point is set at a fixed distance from $x_{3}$;

Two parameters govern this setup : maxb is the maximal value of $b$; valX is the length of $S_{2} . b$ is randomly picked in the interval $[1, \operatorname{maxb}], a$ is drawn in the interval $[1, b]$ and such that $a$ and $b$ are relatively prime, and $\mu$ in the interval [0,2maxb]. The value of $x_{1}$ is drawn in the interval [0, maxb]. The length of $S_{1}$ (i.e. $x_{2}-x_{1}$ ) is randomly selected in the interval [ $\mathrm{valX}, 2 \mathrm{valX}$ ], so that $S_{1}$ is always longer than $S_{2}$. In our test, maxb is set to 1000 and valX varies from 10 to 2 maxb. For each value of valX, 2000 pairs of values $(a, b)$ are drawn. For each of them, 5 different values of $\mu$ are picked up, and then 10 different values of $x_{1}$ are tested, for a total of $10^{5}$ tests.

When the two DSSs are connected, the first test we perform consists in verifying that the three algorithms actually compute the same minimal characteristics. Then, the performances in terms of computation time are compared. Figure 4 shows the results (logarithmic scale for both axis): the $x$-axis represents the value of valX, the $y$-axis is the mean CPU computation time for a pair of DSSs, and for each algorithm.

First we can observe that the experimental behaviour of FastArithmetical algorithm confirms the constant-time complexity. Unsurprisingly, the Arithmetical algorithm has a linear-time complexity. Concerning the StabbingLine algorithm, its performances are slightly worse than the FastArithmetical algorithm, and a slight increase of the mean computation time is observed for larger DSS lengths: this is due to the fact that a post-treatment has to be done on the result returned by this algorithm in order to compute the minimal characteristics. This post-treatment involves a $g c d$ computation, which explains the plot. 


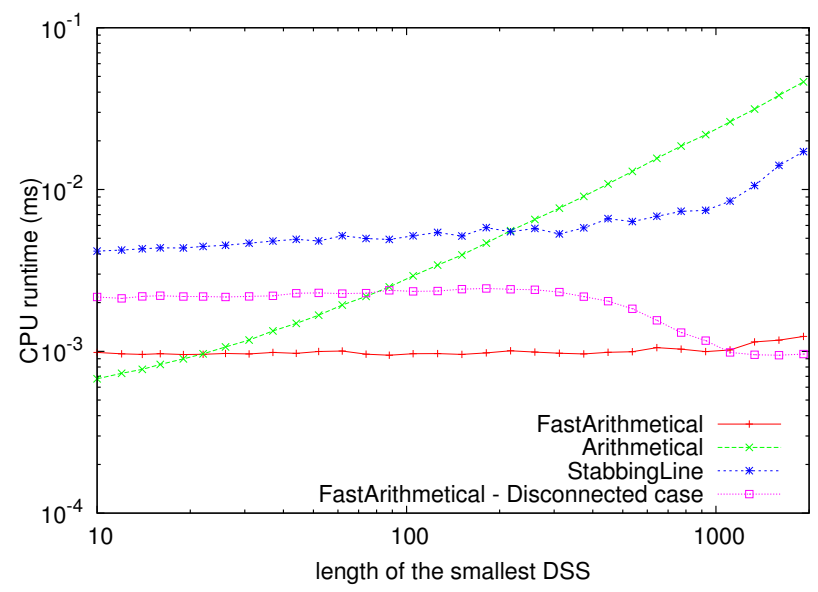

Fig. 5. Experimental results

However, the main information is that the FastArithmetical algorithm gets faster than the classical Arithmetical one when the length of the smallest DSS is greater than 20. In comparison, the StabbingLine algorithm becomes faster for lengths greater than 200 only. This means that what could appear as a small gain on a constant term in comparison to the StabbingLine algorithm makes the FastArithmetical relevant in practice compared to the Arithmetical algorithm. Last, the FastArithmetical remains faster than the StabbingLine algorithm even when the two DSSs are not connected. The slight decrease of the mean computation time for long DSSs is related to the fact that the longer the DSSs, the more easy cases appear.

\section{Conclusion}

In this work, we have shown that the union of two DSSs can be very efficiently computed since it is enough to "update" the minimal characteristics of the first segment with the leaning points of the second one. To do so, we have demonstrated that a state-of-the-art algorithm - the stabbing line algorithm - can be used to compute the union in logarithmic time. Moreover, we have exhibited a number of simple arithmetical properties to design an even faster algorithm. This algorithm runs in $\mathcal{O}(\log (n))$ worst-time complexity, and $\mathcal{O}(1)$ for easy cases and the experiments have shown that the implementation concretises this complexity.

Now, an interesting question remains: what if the union is not possible ? Can we measure the "distance" between the two DSSs ? A solution would be to consider "thicker" DSSs and to compute the thickness necessary for the union to be possible. This problem seems actually to be very close the the blurred DSS recognition algorithms [313, and this trail seems worthy to be followed. 


\section{References}

1. DGtal: Digital Geometry Tools and Algorithms Library, http://libdgtal.org

2. Damiand, G., Coeurjolly, D.: A generic and parallel algorithm for 2D digital curve polygonal approximation. Journal of Real-Time Image Processing (JRTIP) 6(3), $145-157$ (2011)

3. Debled-Rennesson, I., Feschet, F., Rouyer-Degli, J.: Optimal blurred segments decomposition of noisy shapes in linear time. Computers \& Graphics 30(1), 30-36 (2006)

4. Debled-Rennesson, I., Reveillès, J.P.: A linear algorithm for segmentation of digital curves. Inter. Jour. of Pattern Recog. and Art. Intell. 9(6), 635-662 (1995)

5. Debled-Rennesson, I., Reveillès, J.P.: A new approach to digital planes. In: SPIE - Vision Geometry III (1994)

6. Graham, R.L., Knuth, D.E., Patashnik, O.: Concrete Mathematics. AddissonWesley (1994)

7. Harel, D., Tarjan, R.E.: Fast algorithms for finding nearest common ancestor. SIAM Journal on Computing 13(2), 338-355 (1984)

8. Jacob, M.A.: Applications quasi-affines. Ph.D. thesis, Université Louis Pasteur, Strasbourg, France (1993)

9. Lachaud, J.O., Said, M.: Two efficient algorithms for computing the characteristics of a subsegment of a digital straight line. Discrete Applied Mathematics 161(15), 2293-2315 (2013)

10. McIlroy, M.D.: A note on discrete representation of lines. AT\&T Technical Journal 64(2), 481-490 (1985)

11. O'Rourke, J.: An on-line algorithm for fitting straight lines between data ranges. Commun. ACM 24(9), 574-578 (1981)

12. O'Rourke, J.: Computational Geometry in C. Cambridge University Press (1998)

13. Roussillon, T., Tougne, L., Sivignon, I.: Computation of binary objects sides number using discrete geometry, application to automatic pebbles shape analysis. In: Int. Conf. on Image Analysis and Processing, pp. 763-768 (2007)

14. Roussillon, T.: Algorithmes d'extraction de modèles géométriques discrets pour la représentation robuste des formes. Ph.D. thesis, Université Lumière Lyon 2 (2009)

15. Said, M., Lachaud, J.O., Feschet, F.: Multiscale analysis of digital segments by intersection of 2D digital lines. In: ICPR 2010, pp. 4097-4100 (2010)

16. Sivignon, I., Dupont, F., Chassery, J.M.: Digital intersections: minimal carrier, connectiviy and periodicity properties. Graphical Models 66(4), 226-244 (2004)

17. Sivignon, I.: Walking in the farey fan to compute the characteristics of a discrete straight line subsegment. In: Gonzalez-Diaz, R., Jimenez, M.-J., Medrano, B. (eds.) DGCI 2013. LNCS, vol. 7749, pp. 23-34. Springer, Heidelberg (2013) 


\section{Appendix}

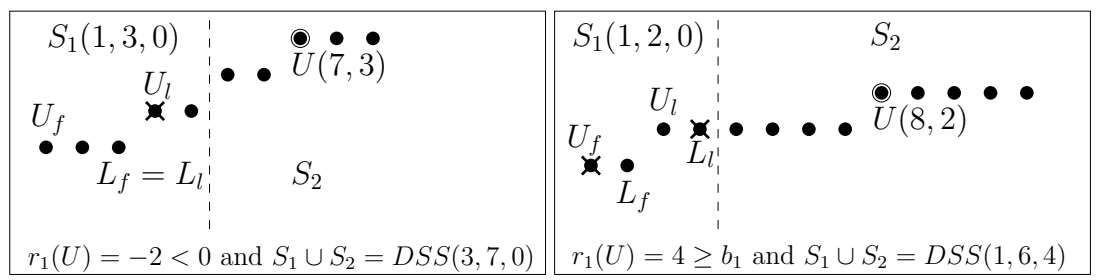

Fig. 6. Illustration of the first line of Table 1 the leaning points of $S_{1}$ marked with a cross cannot be critical support points if the point $U$ of $S_{2}$ is added
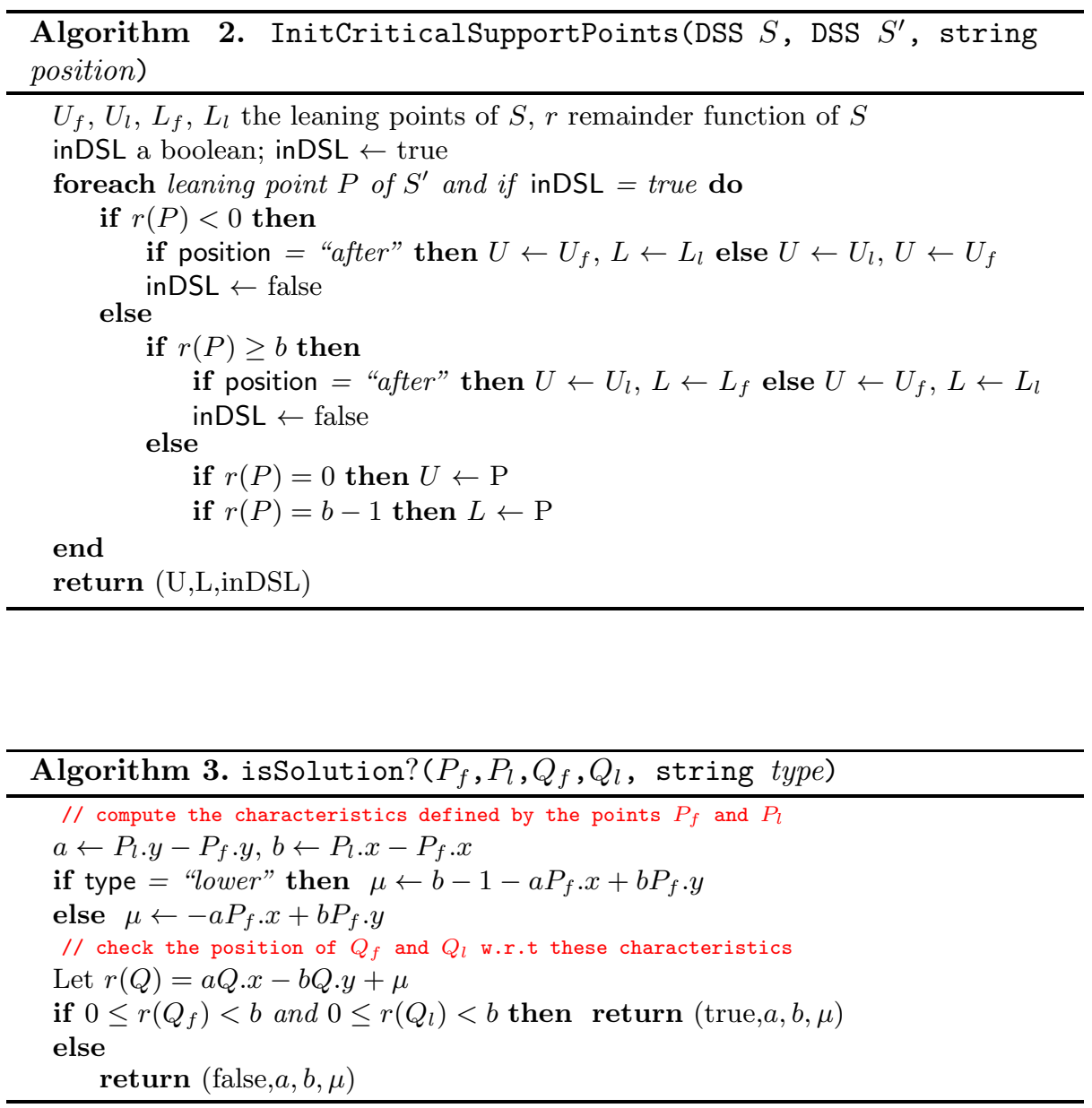